\title{
Seminal Vesicle Mammary-Type Myofibroblastoma
}

National Cancer Institute

\section{Source}

National Cancer Institute. Seminal Vesicle Mammary-Type Myofibroblastoma. NCI

Thesaurus. Code C161640.

An extramammary myofibroblastoma that affects the seminal vesicle. 